\title{
Cholera Toxin Suppresses Expression of Ubiquitin Editing Enzyme A20 and Enhances Transcytosis
}

\author{
Ming-Yang Lij ${ }^{a, d}$ Min Zhu ${ }^{b, d}$ Bing Zhu ${ }^{c, d}$ Zhi-Qiang Wang ${ }^{a}$ \\ aDepartment of Digestive Endoscopy, Division of South Building,Chinese People's Liberation Army \\ General Hospital, Beijing, 'bepartment of Gastroenterology, Division of South Building, Chinese \\ People's Liberation Army General Hospital, Beijing, 'Liver failure treatment and research center; the \\ 302ed Military Hospital, Beijing, dEqually contributed to this work
}

\section{Key Words}

Intestine $•$ Epithelial cells $•$ Barrier function • Ubiquitin editing enzyme A20 • Protein degradation

\begin{abstract}
Background: The ubiquitin editing enzyme A20 plays an important role in maintaining the homeostasis in the body. Microbe-derived adjuvants are commonly used in animal models of intestinal allergy. Objective: This study aims to investigate the role of cholera toxin-induced A20 suppression in compromising intestinal barrier function. Methods: Human intestinal epithelial cells were cultured into monolayers as an in vitro epithelial barrier model. Ovalbumin (OVA) was used as a specific allergen to test the degrading capability of intestinal epithelial cells for the endocytic allergens. The fusion of endosomes and lysosomes in epithelial cells was observed by immunocytochemistry. The antigenicity of OVA was tested by $\mathrm{T}$ cell proliferation assay. Results: A20 was detectable in the intestinal cell lines and mouse intestinal epithelialum. A20 was required in the degradation of endocytic allergens in HT-29 cells. The allergen, OVA, could pass through A20-deficient HT-29 monolayer barrier. Exposure to microbial adjuvant, cholera toxin, suppressed the expression of A20 in HT-29 cells, which compromised the epithelial barrier function. Conclusion: The microbial product, cholera toxin, interferes with the expression of A20 in intestinal epithelial cells, which compromises the intestinal epithelial barrier function.
\end{abstract}

Copyright (c) 2013 S. Karger AG, Basel

\section{Introduction}

The intestinal epithelial barrier consists of the epithelial cell bodies and the tight junctions surrounding the tope of the epithelial cells. The integrity of epithelial barrier is critical in the maintenance of the homeostasis in the body by restricting noxious substances 


\section{Cellular Physiology and Biochemistry}

Cell Physiol Biochem 2013;31:495-504

DOI: 10.1159/000350070

C 2013 S. Karger AG, Basel

www.karger.com/cpb

Li/Zhu/Zhu/Wang: A20 and Epithelial Barrier Function

from absorbing into the tissue [1]. The intestinal epithelial barrier dysfunction is associated with the pathogenesis of a number of chronic immune diseases in the intestine, such as food allergy [2], inflammatory bowel disease [3] and irritable bowel syndrome [4]. The etiology of intestinal epithelial barrier dysfunction is not fully understood yet.

Under the physiological condition, the intestinal epithelial barrier allows the water and some nutrients of small molecular weight to pass freely. The macromolecular protein allergens should be degraded to amino acid or small peptides without the competent antigenicity before being absorbed. However, under allergic condition, the macromolecular allergens do pass across the intestinal epithelial barrier to reach the subepithelial region in the intestine $[5,6]$. The mechanism is not fully understood yet. Two pathways, the paracellular and transcellular pathways, are proposed to mirror the allergens being transported across the intestinal epithelial barrier. The paracellular pathway is sealed by the tight junctions. The transepithelial resistance (TER) in Transwells [7] and conductance in Ussing chambers [8] are indicators of the paracellular pathway states. The increase in allergen transport via the transcellular pathway can be indicated by the increase in the antigen-containing endosomes in the intestinal epithelial cells [9]. Theoretically, the endosomes can fuse with lysosomes in epithelial cells [10]; thus, the endocytic allergens can be degraded by the acidic hydrolases in the lysosomes. However, how the endocytic allergens manage to escape from the machinery of protein degradation in the epithelial cells is unclear.

Cholera toxin (CT) is a protein complex secreted by the bacterium Vibrio cholera. CT is responsible for the massive, watery diarrhea characteristic of the cholera infection. CT can bind to the surface of target cells to be endocytosed into the cytoplasm where it can be wrapped by the plasma membrane to form the endosomes [11]. CT is widely used as an adjuvant in the development of animal models of food allergy $[12,13]$. It is generally considered that microbial products can be used as adjuvants to speed the immune responses in the induction of allergic disease models; but the underlying mechanism is to be further understood.

The ubiquitination is involved in the degradation of protein in the body [14]; A20 is an important ubiquitin editing enzyme, which is also involved in the tethering of endosome and lysosome in the cells [15-17]. The A20-deficient mice suffer from severe immune inflammation in the intestine [18] and some other abnormalities [19] indicating the important role of A20 in the maintenance of the homeostasis in the body. Whether A20 plays a role in the degradation of the endocytic allergens in epithelial cells has not been fully investigated. In this study, we observed that intestinal epithelial cells expressed A20, which played a critical role in the fusion of endosome and lysosome. The microbial product, CT, could suppress A20 in the epithelial cells, which compromised the epithelial barrier function.

\section{Materials and Methods}

Mice

Male BALB/c mice and D011.10 OVA TCR transgenic mice (8-10 week old) were purchased from Lixin Experimental Center (Shanghai, China). The procedures of using animals in this study were approved by the Animal Ethic Committee of the China PLA General Hospital.

\section{Reagents}

The antibodies of A20, CT, OVA, agarose resin, EEA1, LAMP2 and the A20 shRNA were purchased from Santa Cruz Biotech (Shanghai, China). A20 protein was purchased from R\&D Systems (Shanghai, China). CT and OVA protein was purchased from Sigma Aldrich (Shanghai, China). The magnetic cell sorting reagent kits were purchased from Miltenyi Biotech (Shanghai, China). The reagents for real time RT-PCR were purchased from Invitrogen (Shanghai, China). 


\section{Cellular Physiology and Biochemistry}

Cell Physiol Biochem 2013;31:495-504

\begin{tabular}{l|l}
\hline DOI: $10.1159 / 000350070$ & (C) 2013 S. Karger AG, Basel
\end{tabular}

Li/Zhu/Zhu/Wang: A20 and Epithelial Barrier Function

Cell culture

The human colon epithelial cell lines, HT-29 cells (ATCC, HTB-38), T84 cells (ATCC, CCL-248) and Caco-2 cells (ATCC, HTB-37), were cultured in Dulbecco's modified eagle medium/F-12 (DMEM/F12) supplemented with $5 \%$ (vol/vol) fetal calf serum and $2 \mathrm{mM} \mathrm{l-glutamine.} \mathrm{Cells} \mathrm{were} \mathrm{maintained} \mathrm{at} 37^{\circ} \mathrm{C}$ in $5 \% \mathrm{CO}_{2}$. The culture media were changed every day.

Extraction of RNA and protein from the mouse intestinal epithelial cells

Mice were sacrificed by cervical dislocation. A jejunal segment (about $10 \mathrm{~cm}$ ) was excised, opened longitudinally, washed with phosphate buffered saline (PBS). The epithelial tissue was scrapped with a glass slide. The epithelial tissue was then processed for the extraction of RNA and protein following published procedures[17, 20].

\section{Quantitative real-time RT-PCR ( $q R T-P C R$ )}

The cDNA was synthesized from purified total RNA using reverse transcriptase. The primers of the A20 gene are: Forward: gagagcacaatggctgaaca; reverse: tccagtgtgtatcggtgcat (NCBI: NM_006290). The cDNA was amplified using SYBR Green PCR Master Mix on a MyiQ Single Color Real-time PCR Detection System (Bio-Rad Laboratories; Shanghai, China). Each sample was analyzed in triplicate. Data were normalized to a percentage of the internal control $(\beta$-actin).

Enzyme-linked immunoassay (ELISA)

A 96-well plate was coated with sample proteins $(20 \mu \mathrm{g} / \mathrm{ml})$ or the standard proteins (diluted gradedly), or bovine serum albumin (BSA, using as a negative control antigen), and incubated at room temperature for $1 \mathrm{~h}$. The plate was blocked by adding $5 \%$ skim milk and incubated for $1 \mathrm{~h}$. The primary antibody (10 $\mathrm{ng} / \mathrm{ml})$ was added to the plate and incubated for $1 \mathrm{~h}$ and followed by adding the HRP-labeled secondary antibody $(5 \mathrm{ng} / \mathrm{ml})$ to form an immune complex and incubated for $1 \mathrm{~h}$. Washing with TBS was performed after each addition of antibody. The immune complexes were developed by adding tetramethylbenzine; the reaction was stopped by adding $25 \mu \mathrm{l} 2 \mathrm{M} \mathrm{H}_{2} \mathrm{SO}_{4}$. The plate was read with a microplate reader (BioTek, Shanghai, China). The optical density (OD) value of the negative control wells was subtracted from the OD value of each sample well. The contents of target proteins were calculated against the standard curve.

\section{Assessment of epithelial barrier function of HT-29 monolayer}

The transepithelial resistance (TER) and the permeability to OVA of the epithelial monolayers were assessed using as the indicators of the epithelial barrier function. The TER was recorded using an electrovoltohmmeter (World Precision Instruments, Sarasota, FL). The HT-29 monolayer permeability was assessed by determining the allergen (OVA) contents in the basal chambers of the Transwells; the OVA was added to the apical chambers $24 \mathrm{~h}$ before the sampling.

\section{Concentrating proteins in the culture supernatants}

The proteins in the culture supernatants were concentrated by the ammonium sulfate precipitation. Briefly, the culture supernatant was collected and added to $90 \%$ saturation with solid ammonium sulfate, and stired overnight at $4^{\circ} \mathrm{C}$, the precipitate was collected by centrifugation at $4,000 \times \mathrm{g}$ for $10 \mathrm{~min}$ at $4^{\circ} \mathrm{C}$. The precipitate was dissolved in PBS and was desalted by dialyzing through a 4-kDa cutoff dialysis membrane (Sigma) PBS for $24 \mathrm{~h}$. The dialysed suspension was centrifuged at $17,500 \times \mathrm{g}$ for $15 \mathrm{~min}$ at $4^{\circ} \mathrm{C}$. The protein levels in the supernatant were determined by the Bio-Rad protein assay.

Overexpression of A20 in HT-29 cells

The A20 plasmid was a gift from Dr. Guoqing Huang (Shanghai University, China). HT-29 cells were transfected with the plasmid with lipofectamin (Invitrogen, China) following the manufacturer's instruction. The A20 overexpression results were presented in Fig. 4F.

A20 gene silence in HT-29 cells

The A20 gene was knocked down in HT-29 cells with a purchased shRNA reagent kit following the manufacturer's instruction. Some cells were treated with control shRNA using as controls. The A20 gene 


\section{Cellular Physiology and Biochemistry}

Cell Physiol Biochem 2013;31:495-504

\begin{tabular}{l|l}
\hline DOI: $10.1159 / 000350070$ & (c) 2013 S. Karger AG, Basel
\end{tabular}

\begin{tabular}{l|l} 
Publisned OnIIne: Vlarcn 28, 2013 & www.karger.com/cpb
\end{tabular}

Li/Zhu/Zhu/Wang: A20 and Epithelial Barrier Function

knockdown results were presented in Fig. 2. The A20-deficient HT-29 cells could still form monolayers with the TER similar to the control cells.

\section{Immune staining}

The epithelial cells were removed from the supporting filters by the addition of $0.25 \%$ trypsin-EDTA for 5 min. In an Eppendorf tube, HT-29 cells were fixed with 2\% freshly prepared paraformaldehyde for 2 h. After thoroughly washing, the cells were blocked with $1 \%$ BSA (with $0.1 \%$ Triton X-100) for 30 min. The cells were stained with the primary antibodies $(0.5-1 \mu \mathrm{g} / \mathrm{ml})$ for $1 \mathrm{~h}$ at room temperature, washed with PBS and followed by incubating with fluorescence labeled secondary antibodies and washing. The cells were smeared onto a slide, mounted with a cover slip, and observed with a confocal microscope with the objective $\times 630$. The fine structure of the positive staining was further observed with the built-in enlarge feature. The images were photographed when necessary. The slides were observed blindly to avoid the observer bias.

\section{Immunoprecipitation assay and Western blotting}

The epithelial cells were collected from the culture and processed for protein extraction. The extracts were treated with anti-CT and anti-A20 antibodies respectively. The samples were then incubated with protein A-Sepharose. Following incubation, beads were washed twice with immunoprecipitation buffer

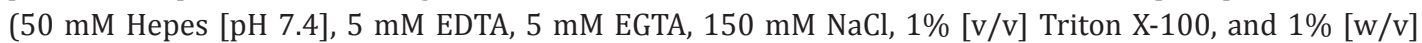
BSA). The proteins were separated by SDS-PAGE (polyacrylamide gel electrophoresis) and transferred onto nitrocellulose membrane. Following the transfer, the the blots on nitrocellulose membrane were blocked with $5 \%$ skim milk for $1 \mathrm{~h}$. Blots were then washed three times with TBST ( $5 \mathrm{~min}$ each), and and incubated with the primary antibodies $(0.5-1 \mu \mathrm{g} / \mathrm{ml})$ overnight at $4 \mathrm{C}$ that followed by incubating with the secondary antibodies (conjugated with horseradish peroxidase) for $1 \mathrm{~h}$ at room temperature. The blots were washed three times with TBST ( 5 min each) after each incubation. The blots were developed with ECL reagent (GE Healthcare).

\section{T cell proliferation assay}

To test the antigenicity of the antigens passed through the epithelial cell monolayers, the $\mathrm{T}$ cell proliferation assay was employed. $\mathrm{CD}^{+} \mathrm{CD}^{+} \mathrm{CD} 25^{-} \mathrm{T}$ cells and dendritic cells (DC) were isolated from spleen cells of OVA-T cell receptor transgenic mice. The mice naturally have T cells expressing the OVAspecific TCR. The T cells were labeled with CFSE (Carboxyfluorescein succinimidyl ester) and cultured with DC ( $\mathrm{T}$ cell:DC $=10: 1)$ in the presence of the extracted proteins $(20 \mu \mathrm{g} / \mathrm{ml})$ for 3 days. The CFSE-dilution assay was performed by flow cytometry.

\section{Statistics}

The data were expressed as mean \pm SD. The difference between two groups was assessed by the Student $t$ test, or ANOVA in three or more groups. $\mathrm{P}<0.05$ was set as the significant criterion.

\section{Results}

Intestinal epithelial cells express A20

We assessed the expression of A20 in intestinal epithelial cells. Total RNA and proteins were extracted from intestinal cell lines, T84 cells, HT-29 cells and Caco- 2 cells, and analyzed by qRT-PCR and Western blotting. As shown in Fig. 1, the expression of A20 was detected in all the three cell lines. To understand if this phenomenon existed in the intestine, we collected epithelial samples from the mouse intestine. Indeed, the expression of A20 was detected in both the small intestine and the colon (Fig. 1).

\section{A20 is required in degradation of endocytic allergens in intestinal epithelial cells}

We next investigated the role of A20 in the degradation of endocytic proteins in the intestinal epithelial cells. HT-29 cells were cultured in the inserts of a Transwell system. After the TER reached $600 \mathrm{Ohm} / \mathrm{cm}^{2}$, a model allergen, OVA, was added to the apical 

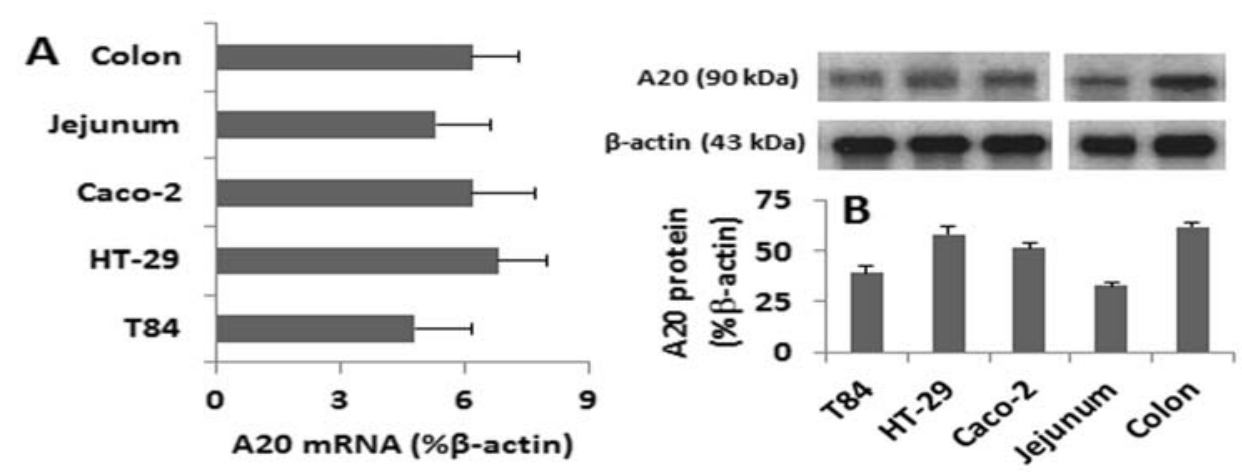

Fig. 1. A20 expression in epithelial cells. The intestinal epithelial cell extracts were processed for detecting the A20 expression. A, the bars indicate the levels of A20 mRNA (assessed by qRT-PCR). B, the immune blots indicate the levels of A20 protein (assessed by Western blotting). The bars below the immune blots indicate the summarized density of the blots. The epithelial samples of jejunum and colon were taken from $\mathrm{BALB} / \mathrm{c}$ mice. The data were presented as mean $\pm \mathrm{SD}$. The experiments were repeated 3 times.

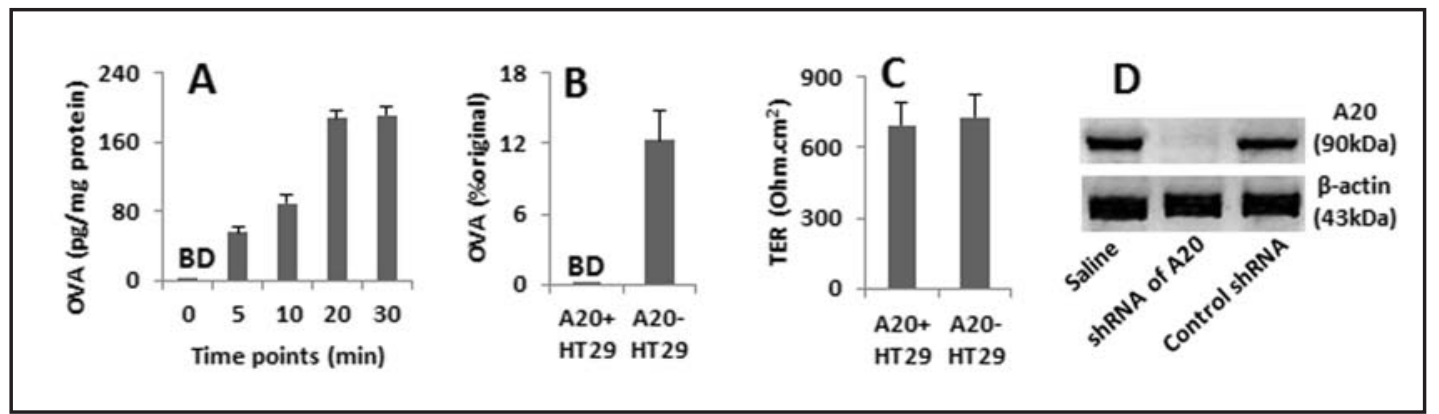

Fig. 2. Assessment of epithelial barrier function. A, the bars indicate the contents of OVA in HT-29 cell extracts. The $\mathrm{X}$ axis shows the time points at which the HT-29 cells were collected. B, the bars indicate the OVA contents in the proteins concentrated from the supernatants of basal chambers. The OVA contents were presented as a percentage of the contents added to the apical chambers. $\mathrm{C}$, the bars indicate the TER recorded at $24 \mathrm{~h}$ after the addition of the OVA. BD: Below the detectable levels. The data were presented as mean \pm SD from 3 separate experiments. D, the immune blots indicate the A20 levels in HT-29 cells treated by saline, or shRNA of A20, or control shRNA.

chambers. The HT-29 monolayers were collected at the time points of 0, 5, 10, 20 and 30 min respectively after the addition of OVA. The cell extracts were analyzed by ELISA. The results showed that the OVA was detected in the cell extracts of HT-29 cells that was increased in a time-dependent manner (Fig. 2A). Samples were also taken from the basal chambers at 30 min and $24 \mathrm{~h}$ later. As analyzed by ELISA, the OVA was not detectable in the basal chambers (Fig. 2B). The results imply that the confluent HT-29 monolayers do not transport antigens with the detectable antigenicity to the basal chambers.

The data in Fig. 2A-B implicate that HT-29 cells can endocytose allergens; the allergens can be degraded in HT-29 cells. Since the ubiquitin editing enzyme A20 is involved in protein degradation in the cell [15-17], we knocked down the A20 gene in HT-29 cells by the gene silence approach. The A20-deficient HT-29 cells still formed monolayers in Transwells. After the TER reached $600 \mathrm{Ohm} \cdot \mathrm{cm}^{2}$, the OVA flux was performed. Abundant OVA was detected in the basal chambers at $24 \mathrm{~h}$ later (Fig. 2B). The results indicate that the A20-deficient HT-29 monolayers can transport OVA from the apical chambers to the basal chambers of Transwells. To elucidate if the transport was via the paracellular pathway, we recorded the TER of the epithelial monolayers at the $24 \mathrm{~h}$ time point. The records showed that the TER did 


\section{Cellular Physiology \\ and Biochemistry}

Cell Physiol Biochem 2013;31:495-504

DOI: 10.1159/000350070

Li/Zhu/Zhu/Wang: A20 and Epithelial Barrier Function

(c) 2013 S. Karger AG, Basel

www.karger.com/cpb
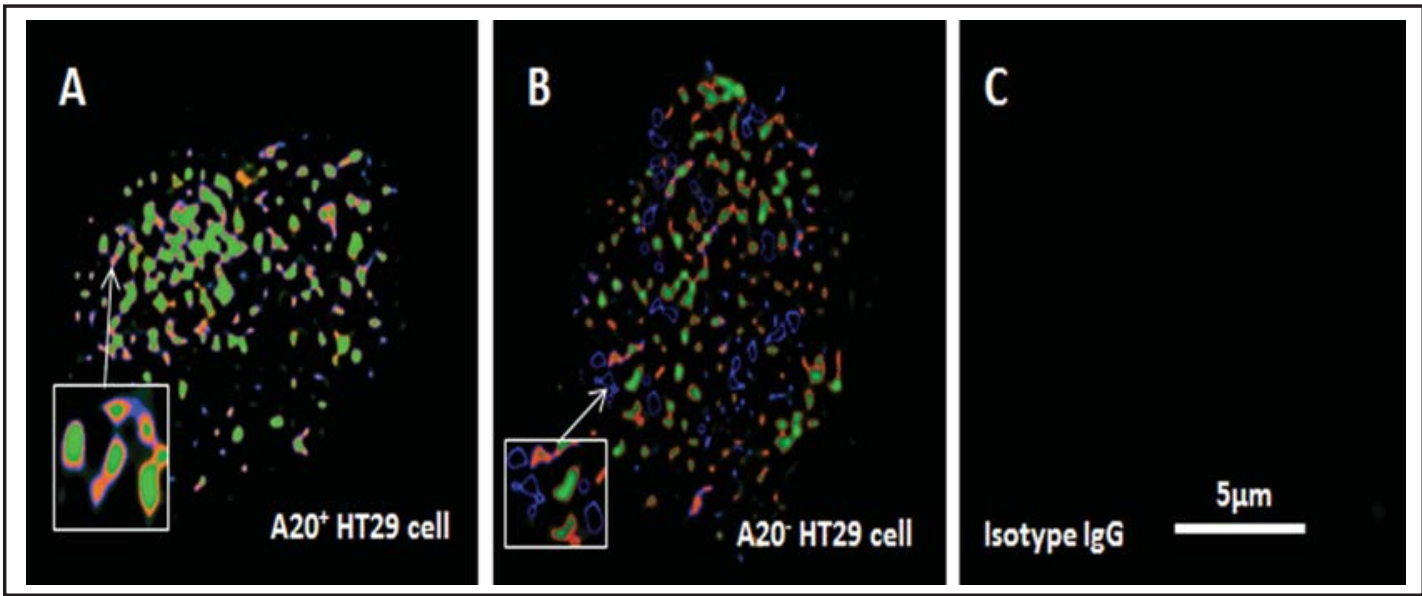

Fig. 3. Fusion of endosome and lysosome. HT-29 cells were exposed to OVA for $30 \mathrm{~min}$ and processed by immunocytochemistry. A-B, the representative confocal images show the positive staining of OVA (green), EEA1 (red; a marker of endosome) and LAMP2 (blue; a marker of lysosome). The inserts are enlarged-images (the original area is pointed by arrows). The original magnification was $\times 630$ (the images were further enlarged with the built-in enlarging feature in the software of the microscope). Each image represents one cell. The experiments were repeated 3 times. $\mathrm{C}$ is an isotype control.

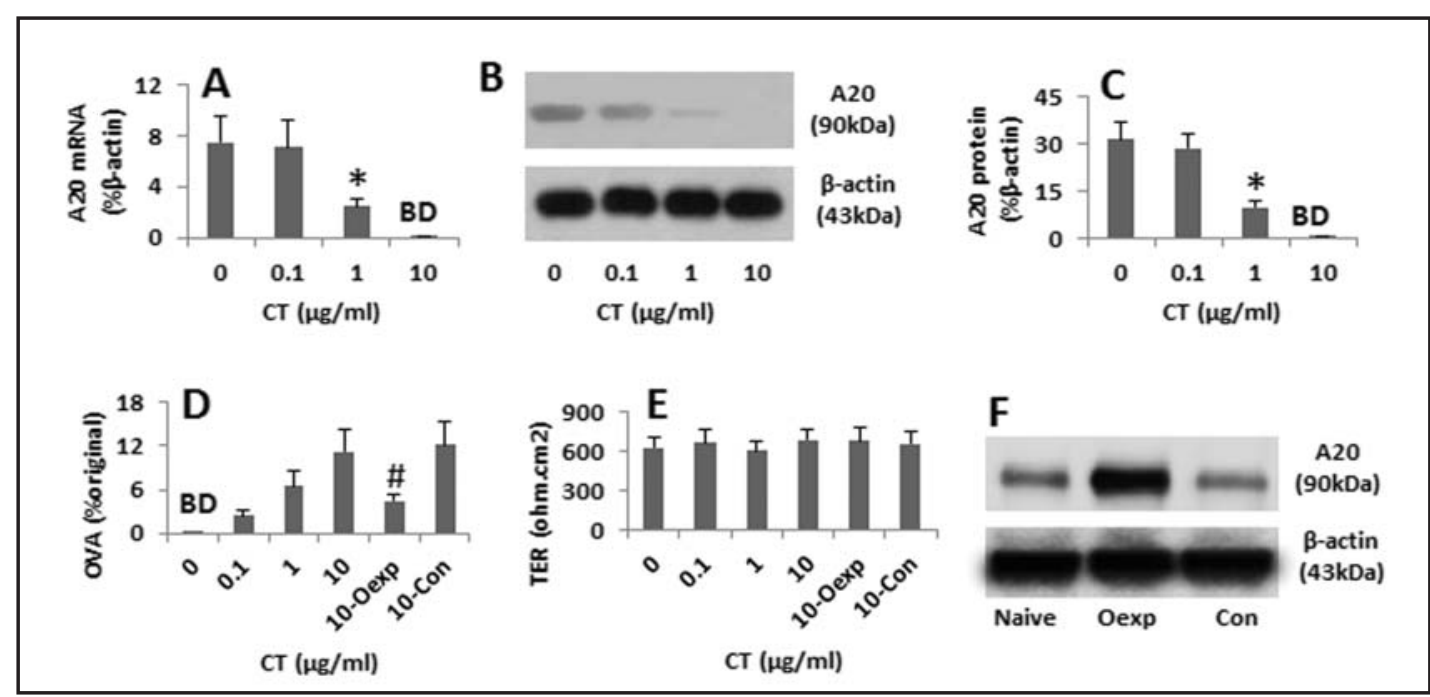

Fig. 4. CT modulates the A20 expression in HT-29 cells. HT-29 cells were stimulated by CT in culture for $24 \mathrm{~h}$. A-B, the bars indicate the levels of A20 mRNA (A; assessed by qRT-PCR) and protein (B; assessed by Western blotting). $C$, the bars indicate the summarized integrated density of the immune blots of $B$. D, the bars indicate the OVA levels in the culture supernatant in the basal chambers of Transwells. E, the bars indicate the TER recorded at $24 \mathrm{~h}$ time point. F, the immune blots indicate the A20 protein. In bar graphs, the $\mathrm{X}$ axis indicate the concentration of CT in the culture medium. Oexp: HT-29 cells with the A20 gene over expression. *, p<0.01, compared with the group "0". \#, p<0.01, compared with dose "10" group.

not drop in the A20-deficient monolayers; instead, the TER was increased about $10 \%$ above the baseline TER that was similar to the naïve controls (Fig. 2C). The data indicate that the transport of OVA to the basal chambers by the A20-deficient HT-29 monolayers is not via the paracellular pathway. 
Fig. 5. Immune complex of A20 and CT. After treating with OVA, or BSA, or CT, in the culture for 48 h, HT-29 cells were collected; proteins were extracted from the cells; the extracts were analyzed by immunoprecipitation assay. The immune blots indicate the complex of CT and A20. IP Ab: Immune precipitation antibody. Plot Ab: Antibodies using in the staining. The data represent 3 separate experiments.

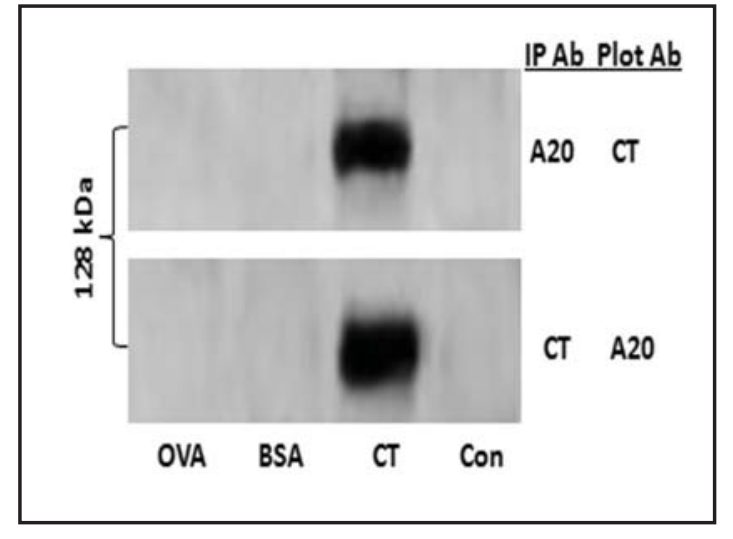

A20 is required in allergen degradation in intestinal epithelial cells

The data in Fig. 2 indicate that HT-29 cells can degrade the endocytic proteins. To take a further insight into the role of A20 in the degradation of endocytic proteins by intestinal epithelial cells, we exposed HT-29 cells to OVA in the culture for $30 \mathrm{~min}$. The cells were collected and observed by confocal microscopy. The $\mathrm{OVA}^{+}$endosomes were observed in the cytoplasm; more than $80 \%$ of the $\mathrm{OVA}^{+}$endosomes were fused with lysosomes while only around 8\% $\mathrm{OVA}^{+}$endosomes were fused with lysosomes in the A20-deficient HT-29 cells (Fig. 3). The results indicate that the $\mathrm{A} 20$ is required in the fusion of endosome/lysosome in HT-29 cells.

Cholera toxin induces intestinal epithelial barrier dysfunction via suppressing A20 expression

Cholera toxin (CT) is one of the adjuvants using in the establishing animal models of intestinal allergy [13]. Mice with induced-intestinal allergy usually have intestinal epithelial barrier dysfunction [21]. To see if CT induces intestinal epithelial barrier dysfunction via interfering with the expression of A20 in the epithelial cells, we exposed HT-29 cells to CT at graded concentrations in culture for $48 \mathrm{~h}$. The results showed that the exposure to CT inhibited the expression of A20 in HT-29 cells in a CT dose-dependent manner. Concurrently exposed HT-29 monolayers to CT and OVA dramatically increased the amounts of OVA in the basal chambers but not altered the TER. Overexpression in HT-29 cells partially antagonized the CT-induced epithelial barrier dysfunction (Fig. 4). The results imply that the exposure to CT compromises the HT-29 monolayer barrier function via suppressing the expression of A20.

A complex of CT and A20 was identified in the epithelial cells

To further look into the mechanism by which the exposure to CT suppressed the expression of A20 in intestinal epithelial cells, HT-29 cells were treated with CT in the culture for $48 \mathrm{~h}$; the cell extracts were analyzed by immune precipitation assay. The results showed an immune complex of CT and A20 was detected in the cell extracts (Fig. 5).

Protein antigens passing through the A20-deficient HT-29 monolayer conserve antigenicity

Data from Fig. 3 indicate that OVA can pass through the A20-deficient HT-29 monolayer to reach the basal chambers. We wondered if the OVA in the basal chambers still conserved the antigenicity. We isolated the $\mathrm{CD}^{+} \mathrm{CD}^{+} \mathrm{CD} 25^{-} \mathrm{T}$ cells from the spleen of D011.10 mice; the T cells were cultured in the presence of DCs and the concentrated proteins $(10 \mu \mathrm{g} / \mathrm{ml})$ from the basal chambers for 3 days. As shown by CFSE-dilution assay, proteins from the A20deficient group or CT-treated HT-29 cell group induced marked T cell proliferation, the latter was partially antagonized by the A20 gene overexpression; proteins from the A20-sufficient group did not induce detectable T cell proliferation (Fig. 6). The results indicate that proteins passing through the A20-deficient HT-29 monolayers still conserve the antigenicity. 


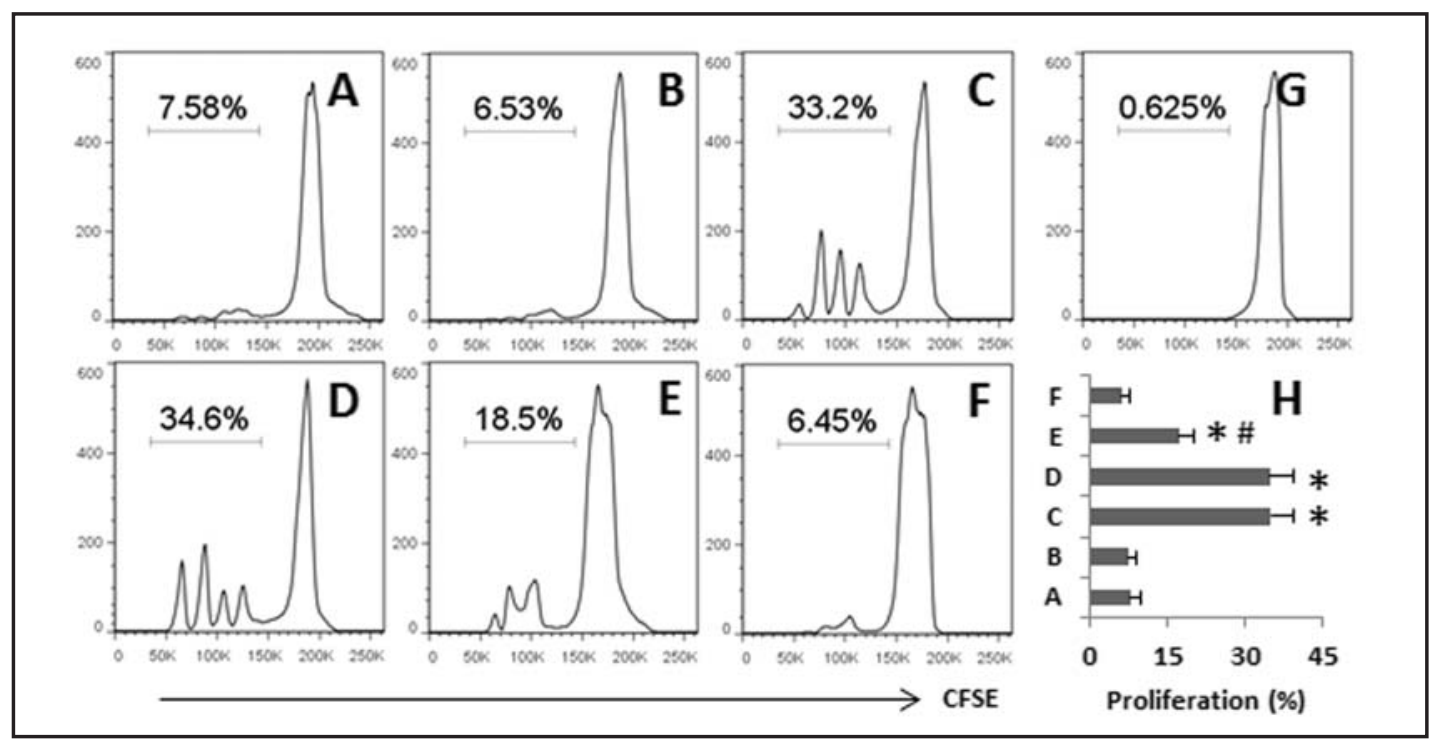

Fig. 6. T cell proliferation. $\mathrm{CD}^{+} \mathrm{CD}^{+}{ }^{+} \mathrm{CD} 25^{-} \mathrm{T}$ cells and $\mathrm{DC}$ were isolated from D011.10 mouse spleen. The $\mathrm{T}$ cells and DC $(10: 1)$ were cultured in the presence of medium alone (A), protein extracts from culture supernatants (B-E) of naïve HT-29 cells (B), or A20-deficient HT-29 cells (C), or CT-treated HT-29 cells (D), or CT-treated A20-overexpressing HT-29 cells (E), or BSA-treated HT-29 cells. The histograms indicate the T cell proliferation (the gated cells). (G) is a CFSE staining control. (H), the bars indicate the summarized data of panels A-E. *, p<0.01, compared with group A. The data represent 3 separate experiments.

\section{Discussion}

The present data indicate that A20 plays an important role in maintaining the epithelial barrier function. The data showed that A20 was required in the fusion of endosome and lysosome in HT-29 cells. The deficiency of A20 resulted in that the endocytic allergens were not degraded in HT-29 cells, which were transported to the basal chambers of the Transwells. The study also revealed that the exposure to CT resulted in the suppression of A20 expression in HT-29 cells that further induced the HT-29 monolayer barrier dysfunction.

The ingested food contains plenty of allergens. These allergens can be degraded to amino acids or small peptides by the digestive enzymes in the gastrointestinal tract. There may be still a small quantity of allergens with antigenicity to be absorbed into the intestinal epithelial cells. The epithelial cells are supposed to further degrade the endocytic allergens to minimize the antigenic proteins to be transported to the subepithelial region to avoid inducing unwanted immune responses. The present data are in line with the information by showing that no detectable antigenic proteins are transported to the basal chambers of the Transwells.

After endocytosis, the endocytic proteins are wrapped by the plasma membrane to form endosomes. The endosomes then fuse with lysosomes; the endocytic proteins are degraded by the acid hydrolases in the lysosomes. Our results also showed that the endocytic OVA was wrapped with the plasma membrane, which was EEA1 positive indicating these particles are endosomes. More than $80 \%$ endosomes were fused with lysosomes in normal epithelial cells as shown by the present data. In line with the previous reports $[16,17]$, the present data also show that the A20 is required in the fusion of endosome and lysosome in HT-29 cells. The deficiency of A20 resulted in that only about $5 \%$ endosomes were fused with lysosomes. The results have mirrored the importance of A20 in the degradation of endocytic allergens in epithelial cells. Others also propose that A20 is important in the tethering of endosome and lysosome [15-17]. 


\section{Cellular Physiology and Biochemistry}

Cell Physiol Biochem 2013;31:495-504

DOI: $10.1159 / 000350070$

C 2013 S. Karger AG, Basel

www.karger.com/cpb

Li/Zhu/Zhu/Wang: A20 and Epithelial Barrier Function

The major role of the intestinal epithelial barrier is to restrict the noxious substances from absorbing into the intestinal mucosa. One of the indicators of the intestinal epithelial barrier dysfunction is macromolecular substances to be transported across the epithelial barrier. Such a phenomenon was observed in the present study; abundant OVA with detectable antigenicity was transported to the basal chambers of the Transwells in the A20-deficient HT-29 monolayers. Our results showed that the OVA transport was via the transcellular pathway; it is less likely via the paracellular pathway since the TER of the HT-29 monolayers were not altered.

CT has been used for years as an adjuvant in the establishing food allergy animal models via stimulating a Th2 response and the production of IgG1 antibodies [22, 23]. Our data have added novel information on this topic by showing that CT can bind to A20 to form complexes and suppress the expression of A20 in the HT-29 cells that resulted in the epithelial barrier dysfunction. It is noteworthy that CT-induced barrier dysfunction manifests the increase in the allergen transcellular transport; even in the states that the paracellular pathway is not disturbed.

How CT disturbs the fusion of endosome and lysosome in epithelial cells is unclear. Our data indicate that after the addition of CT to the epithelial culture, the expression of A20 was decreased at both gene transcriptional level and protein level. Further evidence indicates that CT can bind to A20 protein in the epithelial cells. Subsequently, the complexes of CT/ A20 may be cleaned by the cells, which needs further investigated.

In summary, the present study indicate that A20 plays a critical role in the fusion of endosome and lysosome in intestinal epithelial cells. Exposure to microbial product, CT, can suppress the expression of A20 in epithelial cells, which further compromises the epithelial barrier function.

\section{References}

1 Hoque KM, Chakraborty S, Sheikh IA, Woodward OM: New advances in the pathophysiology of intestinal ion transport and barrier function in diarrhea and the impact on therapy. Expert Rev Anti Infect Ther 2012;10:687-699.

2 Yu LC: Intestinal epithelial barrier dysfunction in food hypersensitivity. J Allergy (Cairo) 2012;2012:596081.

-3 Watson AJM, Hughes KR: TNF-alpha induced intestinal epithelial cell shedding: implications for intestinal barrier function. Ann N Y Acad Sci 2012;1258:1-8.

4 Ohman L, Simren M: Pathogenesis of IBS: role of inflammation, immunity and neuroimmune interactions. Nat Rev Gastroenterol Hepatol 2010;7:163-173.

-5 Tu YH, Oluwole C, Struiksma S, Perdue MH, Yang PC: Mast cells modulate transport of CD23/IgE/antigen complex across human intestinal epithelial barrier. N Am J Med Sci 2009;1:16-24.

-6 Yu LCH, Yang PC, Berin MC, Di Leo V, Conrad DH, McKay DM Satoskar AR, Perdue MH: Enhanced Transepithelial Antigen Transport in Intestine of Allergic Mice Is Mediated by IgE/CD23 and Regulated by Interleukin-4. Gastroenterology 2001;121:370-381.

7 Kreisberg RB, Harper J, Strauman MC, Marohn M, Clements JD, Nataro JP: Induction of Increased Permeability of Polarized Enterocyte Monolayers by Enterotoxigenic Escherichia coli Heat-Labile Enterotoxin. Am J Trop Med Hyg 2011;84:451-455.

-8 Yang PC, Jury J, Soderholm JD, Sherman PM, McKay DM, Perdue MH: Chronic Psychological Stress in Rats Induces Intestinal Sensitization to Luminal Antigens. Am J Pathol 2006;168:104-114.

-9 Berin MC, Kiliaan AJ, Yang PC, Groot JA, Taminiau JA, Perdue MH: Rapid transepithelial antigen transport in rat jejunum: impact of sensitization and the hypersensitivity reaction. Gastroenterology 1997;113:856864.

10 Bröcker C, Engelbrecht-Vandré S, Ungermann C: Multisubunit Tethering Complexes and Their Role in Membrane Fusion. Curr Biol 2010;20:R943-R952.

11 Sandvig K, Spilsberg B, Lauvrak SU, Torgersen ML, Iversen TG, van Deurs B: Pathways followed by protein toxins into cells. Int J Med Microbiol 2004;293:483-490. 


\section{Cellular Physiology and Biochemistry}

Cell Physiol Biochem 2013;31:495-504

\begin{tabular}{l|l}
\hline DOI: 10.1159/000350070 & (C) 2013 S. Karger AG, Basel
\end{tabular}

Li/Zhu/Zhu/Wang: A20 and Epithelial Barrier Function

12 Aldemir H, Bars R, Herouet-Guicheney C: Murine models for evaluating the allergenicity of novel proteins and foods. Regul Toxicol Pharmacol 2009;54:S52-S57.

-13 Bailón E, Cueto-Sola M, Utrilla P, Rodríguez-Ruiz J, Garrido-Mesa N, Zarzuelo A, Xaus J, Gálvez J, Comalada M: A shorter and more specific oral sensitization-based experimental model of food allergy in mice. J Immunol Methods 2012;381:41-49.

14 Yamaguchi H, Hsu J, Hung M: Regulation of ubiquitination-mediated protein degradation by survival kinases in cancer. Front Oncol 2012;2:15.

-15 Li L, Soetandyo N, Wang Q Ye Y: The zinc finger protein A20 targets TRAF2 to the lysosomes for degradation. Biochim Biophys Acta 2009;1793:346-353.

16 Song CH, Liu ZQ, Huang S, Zheng PY, Yang PC: Probiotics promote endocytic allergen degradation in gut epithelial cells. Biochem Biophys Res Commun 2012;426:135-140.

17 Chen C, Yang G, Geng XR, Wang X, Liu Z, Yang PC: TNFAIP3 Facilitates Degradation of Microbial Antigen SEB in Enterocytes. PLoS ONE 2012;7:e45941.

18 Vereecke L, Sze M, Guire CM, Rogiers B, Chu Y, Schmidt-Supprian M Pasparakis M, Beyaert R, van Loo G: Enterocyte-specific A20 deficiency sensitizes to tumor necrosis factor-induced toxicity and experimental colitis. J Exp Med 2010;207:1513-1523.

19 Hövelmeyer N, Reissig S, Xuan NT, Adams-Quack P, Lukas D, Nikolaev A, Schlüter D, Waisman A: A20 deficiency in B cells enhances B-cell proliferation and results in the development of autoantibodies. Eur J Immunol 2011;41:595-601.

20 Mahmood T, Yang PC: Western blot: technique, theory, and trouble shooting. N Am J Med Sci 2012;4:429434.

21 Bol-Schoenmakers M, Bleumink R, Marcondes Rezende M, Mouser E, Hassing I, Ludwig I, Smit JJ, Pieters RH: Diclofenac enhances allergic responses in a mouse peanut allergy model. Clin Exp Allergy 2011;41:424-433.

22 Marinaro M, Staats HF, Hiroi T, Jackson RJ, Coste M, Boyaka PN, Okahashi N, Yamamoto M, Kiyono H, Bluethmann H, Fujihashi K, McGhee JR: Mucosal adjuvant effect of cholera toxin in mice results from induction of T helper 2 (Th2) cells and IL-4. J Immunol 1995;155:4621-4629.

23 Snider DP, Marshall JS, Perdue MH, Liang H: Production of IgE antibody and allergic sensitization of intestinal and peripheral tissues after oral immunization with protein $\mathrm{Ag}$ and cholera toxin. J Immunol 1994;153:647-657. 